\title{
Tycoon: an Implementation of a Distributed, Market-based Resource Allocation System
}

\author{
Kevin Lai Lars Rasmusson Eytan Adar Stephen Sorkin Li Zhang Bernardo A. Huberman \\ \{klai, lars.rasmusson, eytan.adar, stephen.sorkin, l.zhang, bernardo.huberman\}@hp.com
}

HP Labs Palo Alto

October 31, 2018

\begin{abstract}
Distributed clusters like the Grid and PlanetLab enable the same statistical multiplexing efficiency gains for computing as the Internet provides for networking. One major challenge is allocating resources in an economically efficient and low-latency way. A common solution is proportional share, where users each get resources in proportion to their pre-defined weight. However, this does not allow users to differentiate the value of their jobs. This leads to economic inefficiency. In contrast, systems that require reservations impose a high latency (typically minutes to hours) to acquire resources.

We present Tycoon, a market based distributed resource allocation system based on proportional share. The key advantages of Tycoon are that it allows users to differentiate the value of their jobs, its resource acquisition latency is limited only by communication delays, and it imposes no manual bidding overhead on users. We present experimental results using a prototype implementation of our design.
\end{abstract}

\section{Introduction}

A key advantage of distributed systems like the Grid [13] and PlanetLab [1] is their ability to pool together shared computational resources. This allows increased throughput because of statistical multiplexing and the bursty utilization pattern of typical users. Sharing nodes that are dispersed in the network allows lower delay because applications can store data close to users. Finally, sharing allows greater reliability because of redundancy in hosts and network connections.

The key issue for shared resources is allocation. One solution is to add more capacity. If resources are already optimally allocated, then this is the only solution, albeit a costly one. In all other cases, allocation and additional capacity are complementary. In addition, in peer-to-peer systems where organizations both consume and provide resources (e.g.,
PlanetLab), careful allocation can effectively increase capacity by providing assurances to reluctant organizations that contributions will be returned in kind.

However, resource allocation remains a difficult problem. The key challenges for resource allocation in distributed systems are: strategic users who act in their own interests, a rapidly changing and unpredictable demand, and hundreds or thousands of unreliable hosts that are physically and administratively distributed.

Our approach is to incorporate an economic mechanism [16] (e.g., an auction) into the resource allocation system. Systems without such mechanisms 29. 6] 34 typically assume that task values (i.e., their importance) are the same, or are inversely proportional to the resources required, or are set by an omniscient administrator. However, in many cases, task values vary significantly, are not correlated to resource requirements, and are difficult and time-consuming for an administrator to set. Instead, market-based resource allocation systems [31. 10, 33, 5] rely on users to set the values of their own jobs and provide a mechanism to encourage users to truthfully reveal those values.

Despite these advantages, we are not aware of any currently operational market-based resource allocation systems for computational resources. We believe one key impediment is that previously proposed systems impose a significant burden on users: frequent interactive bidding, or, conversely, infrequent bidding that increases the latency to acquire resources. Most users would prefer to run their program as they would without a market-based system and forget about it until it is done. The latency to acquire resources is important for applications like a web server that needs to allocate resources quickly in reaction to unexpected events (e.g., breaking news stories from $\mathrm{CNN}$ ). In addition, many market-based systems rely on a centralized market that limits reliability and scalability. 
In this paper, we present the Tycoon distributed, market-based resource allocation system. Each providing Tycoon host runs an auctioneer process that multiplexes the local physical resources for one or more virtual hosts (using Linux VServers [2]). As a result, if an auctioneers fails, users can still acquire resources at other hosts. Clients request resources from auctioneers using continuous bids that can be as infrequent as the user wishes while still allowing immediate acquisition of resources.

The contribution of this paper is the design, implementation, and evaluation of Tycoon. We describe a prototype implementation of our design running on a 22-host cluster distributed between Palo Alto in California and Bristol in the United Kingdom. Tycoon can reallocate all of the hosts in this cluster in less than 30 seconds. We show that Tycoon encourages efficient usage of resources even when users make no explicit bids at all. We show that Tycoon provides these benefits with little overhead. Running a typical task on a Tycoon host incurs a less than a $5 \%$ overhead compared to an identical non-Tycoon host. Using our current modest server infrastructure (450 $\mathrm{MHz}$ x86 CPU, $100 \mathrm{MB} / \mathrm{s}$ Ethernet), limited tests indicate that our current design scales to 500 hosts and 24 simultaneous active users (or any other combination with a product of 12,000 ). The main limitation of this implementation is that it only manages CPU cycles (not memory, disk, etc.), but we expect to resolve this by upgrading the virtualization software.

The paper is organized as follows. In $\S 2$ we give an overview of the Tycoon design. In $\S 3$, we describe the Tycoon architecture in detail. In $\S 4$ we present the results of experiments using the Tycoon system. In $\oint 5$, we review related work in resource allocation. We describe some extensions to the basic design in $\S[$ and conclude in $\S$ [7

\section{Design Overview}

In this section, we present the service model and interface that Tycoon provides to users. We describe the architecture of Tycoon in more detail in $\S$ [3.

\subsection{Service Model Abstraction}

The purpose of Tycoon is to allocate compute resources like CPU cycles, memory, network bandwidth, etc. to users in an economically efficient way. In other words, the resources are allocated to the users who value them the most. To give users an incentive to truthfully reveal how much they value resources, users use a limited budget of credits to bid for resources. The form of a bid is $(h, r, b, t)$, where $h$ is the host to bid on, $r$ is the resource type, $b$ is the number of credits to bid, and $t$ is the time interval over which to bid. This bid says, "I'd like as much of $r$ on $h$ as possible for $t$ seconds of usage, for which I'm willing to pay $b$ ". This is a continuous bid in that it is in effect until cancelled or user runs out of money.

The user submits this bid to the auctioneer that runs on host $h$. This auctioneer calculates $b_{i}^{r} / t_{i}^{r}$ for each bid $i$ and resource $r$ and allocates its resources in proportion to the bids. This is a "best-effort" allocation in that the allocation may change as other bids change, applications start and stop, etc. Credits are not spent at the time of the bid; the user must utilize the resource to burn the credits. To do this, a user uses ssh to run a program. The $t$ seconds of usage can be used immediately or later and at the same time or in pieces, as the user wishes.

Note that the auctioneers are completely independent and do not share information. As a result, if a user requires resources on two separate hosts, it is his responsibility to send bids to those two markets. Also, markets for two different resources on the same host are separate.

This service model has two advantages. First, the continuous bid allows user agents to express more sophisticated preferences because they can place different bids in different markets. Specific auctioneers can differentiate themselves in a wide variety of ways. For example, an auctioneer could have more of a resource (e.g. more CPU cycles), better quality-ofservice (e.g., a guaranteed minimum number of CPU cycles), a favorable network location, etc. A user agent can compose bids however it sees fit to satisfy user preferences. Second, since the auctioneers push responsibility for expressing sophisticated bids onto user agents, the core infrastructure can remain efficient, scalable, secure, and reliable. The efficiency and scalability are a result of using only local information to manage local resources and operating over very simple bids. The security and reliability are a result of independence between different auctioneers.

\section{$2.2 \quad$ Interface}

In this section, we describe how a user uses the system. The interface requirments are important because we believe the bidding requirements of previous economic systems were burdensome for users.

Table 2.2 lists the main Tycoon user commands. These are currently implemented as a Linux command-line tool, but they could easily be implemented in a graphical user interface. The first action 


\begin{tabular}{|l|l|}
\hline Command & Action \\
\hline \hline tycoon create_account host0 10 10 10 & $\begin{array}{l}\text { Create an account on host0 with a bid of 10 initial credits } \\
\text { for CPU cycles, memory, and disk. }\end{array}$ \\
\hline tycoon fund host0 cpu 901000 & $\begin{array}{l}\text { Fund the account on host0 using 90 credits to be spent } \\
\text { over } 1000 \text { seconds for CPU cycles. }\end{array}$ \\
\hline tycoon set_interval host0 cpu 2000 & Change bid interval on the account to 2000 for CPU cycles. \\
\hline tycoon get_status host0 & $\begin{array}{l}\text { Get status of account including the current balance, cur- } \\
\text { rent interval, etc. for each of the resources. }\end{array}$ \\
\hline
\end{tabular}

Table 1: This table shows the main Tycoon user commands.

a user takes is to create an account on a providing host. This notifies auctioneers that a user intends to bid on that host and makes an initial bid. The bid interval defaults to $10,000,000$ seconds so that the user is unlikely to run out of money. Account creation only needs to be done rarely (in most cases once) per user and host. Users usually perform account creation, like the operations that follow, on many hosts, so the command-line tool allows the same operation to be performed on multiple hosts in parallel.

At this point, the user can ssh into hosts and run his application. Users are not required to change their bids when they start and stop tasks. They can do so to optimize their resource usage, if they wish. However, the auctioneers will still deduct credits when he runs. As a result, users who run infrequently will get more resources than those who run continuously. If the user chooses, he can transfer more money to his account and/or change the bidding interval. He might have a critical task for which he is willing to spend credits at a higher rate, or, conversely, he might have a very low priority job, for which he wishes to decrease his spending rate. The key point is that the users are relieved from any mandatory interaction with the system.

\section{Architecture}

Tycoon is split into the following components: service location service (SLS), bank, auctioneer, and agent. The design of the SLS and bank are not novel, but we describe them here because they are necessary components for a working implementation.

\subsection{Service Location Service}

Auctioneers use the service location service to advertise resources, and agents use it to locate resources (as shown in steps 1 and 2 in Figure 11). Our prototype uses a simple centralized soft-state server, but the other components would work just as well with more sophisticated and scalable service location systems (e.g., Ganglia [19 and SWORD 21]). Auctioneers register their status with the SLS every 30 seconds and the SLS de-registers any auctioneer that has not contacted it within the past 120 seconds. This status consists of the total amount bid on the host for each resource, the total amount of each resource type available (e.g., CPU speed, memory size, disk space), etc. The status is cryptographically signed by the auctioneer and includes the auctioneer's public key. Clients store this key and use it to authenticate the results of later queries and also to authenticate direct communications with the auctioneer.

The soft-state design allows the system to be robust against many forms of hardware and software failures. The querying agents may receive stale information from the SLS, but they will receive updated information if they elect to contact an auctioneer directly.

\subsection{Bank}

The bank maintains account balances for all users and providers. Its main task is to transfer funds from a client's account to a provider's account (shown in step 3 in Figure (1).

We assume that the bank has a well-known public key and that the bank has the public keys of all the users. These are the same requirements for any user to securely use a host with or without a marketbased resource allocation system. We further assume roughly synchronized clocks. In describing the transfer protocol, we use Alice and Bob as the fictional example sender and receiver. Alice begins by sending a message to the bank as follows:

$$
\text { Alice, Bob, amount, time, }
$$

$$
\text { Sign }_{\text {Alice }} \text { (Alice, Bob, amount, time) }
$$

Sign $_{\text {Alice }}$ is the DSA signature function using Alice's private key. The bank verifies that the signature is correct, which implies that the message is from Alice, 


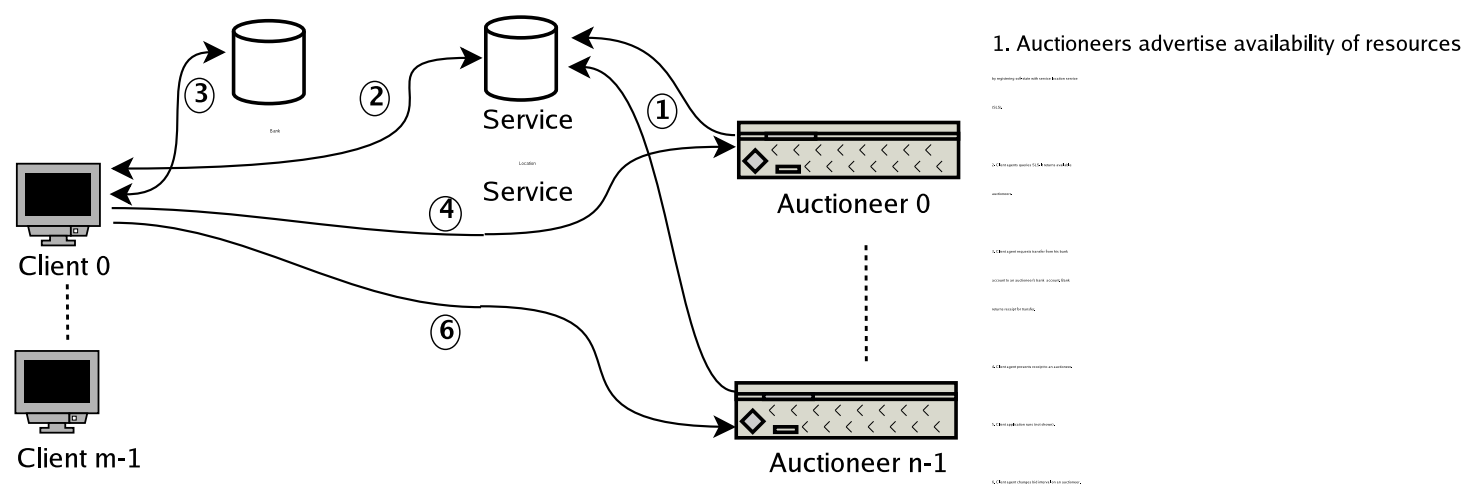

Figure 1: This figure gives an overview of how the Tycoon components interact.

that the funds are for Bob, and that the amount and time are as specified. The bank keeps a list of recent messages and verifies that this message is new, thus guarding against replay attacks. Assuming this is all correct and the funds are available, the bank transfers amount from Alice to Bob and responds with the following message (the receipt):

$$
\begin{gathered}
\text { Alice, Bob, amount, time, } \\
\operatorname{Sign}_{\text {Bank }}(\text { Alice, Bob, amount, time })
\end{gathered}
$$

The bank sends the same time as in the first message. Alice verifies that the amount, time, and recipient are the same as the original message and that the signature is correct. Assuming the verification is successful, Alice forwards this message to Bob as described in $\S 3.3$ Bob keeps a list of recent receipts and verifies that this receipt is new, thus guarding against replay attacks.

The advantages of this scheme are simplicity, efficiency, and prevention of counterfeiting. Microcurrency systems are generally complex, have high overhead, and only discourage counterfeiting. The disadvantages of this approach are scalability and vulnerability to compromise of the bank. However, bank operations are relatively infrequent (see $\S 3.3 .2$ for how bids can be changed without involving the bank), so scalability is not a critical issue for moderate numbers of users and hosts, as we show in $\S 4.4$ The vulnerability to compromise of the bank could be a problem and we discuss possible solutions in $\S[$ 目

\subsection{Auctioneer}

Auctioneers serve four main purposes: management of local resources, collection of bids from users, allocation of resources to users according to their bids, and advertisment of the availability of local resources.

\subsubsection{Virtualization}

To manage resources, an auctioneer relies on a virtualization system and a local allocation system. Our implementation uses Linux VServer (with modifications from PlanetLab) for virtualization. VServer provides each user with a separate file system and gives the appearance that he is the sole user of a machine, even if the physical hardware is being shared. The user accesses this virtual machine by using ssh.

VServers virtualize at the system call level, which provides the advantage of low overhead. We show in $\S 4.3$ that the total auctioneer overhead, including VServers, is at most ten percent and usually much less. Systems that virtualize at the hardware level like VMWare 3] or Disco [7] have significantly more overhead [12].

For local allocation, Tycoon uses the plkmod proportional share scheduler [6], which implements the standard proportional share scheduling abstraction [28. The disadvantage of VServers and plkmod is that they do not completely virtualize system resources. This is why Tycoon currently only manages CPU cycles. In $\S$ 6 we discuss new virtualization and allocation systems that provide this functionality.

\subsubsection{Setting Bids}

The second purpose of auctioneers is to collect bids from users. Auctioneers store bids as two parts for each user: the local account balance, and the bidding interval. The local balance is the amount of money the user has remaining locally. The bidding interval specifies the number of seconds over which to spend the local balance. Users have two methods of changing this information: fund and set_interval. fund 
transfers money from the user's bank account to the auctioneer's bank account, and conveys that fact to the auctioneer. It has the disadvantage that it requires significant latency $(100 \mathrm{~ms})$ and it requires communication with the bank, which may be offline or overloaded. set_interval sets the bidding interval at the auctioneer without changing the local balance. It only requires direct communication between the client and the auctioneer, so it provides a low latency method of adjusting the bid until the local balance is exhausted.

In describing the fund protocol, we again use Alice and Bob as examples. We assume that Alice and Bob already have each other's public keys and that Alice has the value nonce $_{\text {Alice }}$. A nonce is a unique token which Bob has never seen from Alice before. In the current implementation it is an increasing counter. First, Alice gets a bank receipt as described above. She then sends the following message to Bob:

$$
\begin{gathered}
\text { Alice, Bob, } \text { nonce }_{\text {Alice }}, \text { interval, receipt, } \\
\operatorname{Sign}_{\text {Alice }}\left(\text { Alice, Bob, } \text { nonce }_{\text {Alice }}, \text { interval, receipt }\right)
\end{gathered}
$$

The nonce allows Bob to detect replay attacks. Bob verifies that he is the recipient of this message, that the nonce has not been used before, that the receipt specifies that Alice has transferred money into his account, that the bank has correctly signed the receipt, and that Alice has correctly signed this message. Assuming this is all correct, Bob increases Alice's local balance by the amount specified in the receipt and sets Alice's bidding interval to interval. set_interval is identical, except that it does not include the bank receipt.

The key advantage of separating fund and set_interval is that it reduces the frequency of bank operations. Users only have to fund their hosts when they wish to change the set of hosts they are running on or when they receive income. For most users and applications, we believe this is on the order of days, not seconds. Between fundings, users can modify their bids by changing the bidding interval, as described in the next section.

\subsubsection{Allocating Resources}

The third and most important purpose of auctioneers is to use virtualization and the users' bids to allocate resources among the users and account for usage. Although our current implementation only allocates CPU cycles because of virtualization limitations, the following applies to both rate-based (e.g., CPU cycles and network bandwidth) and spacebased (e.g., physical memory and disk space) re- sources. In addition, we initially describe a proportional share-based function, but there are other allocation functions with desirable properties (e.g., Generalized Vickrey Auctions, described below).

For each user $i$, the auctioneer knows the local balance $b_{i}$ and the bidding interval $t_{i}$. The auctioneer calculates the bid as $b_{i} / t_{i}$. Consider a resource with total size $R$ (e.g., the number of cycles per second of the CPU or the total disk space) over some period $P$. The allocation function for $r_{i}$, the amount of resource allocated to user $i$ over $P$, is

$$
r_{i}=\frac{\frac{b_{i}}{t_{i}}}{\sum_{j=0}^{n-1} \frac{b_{j}}{t_{j}}} R .
$$

Let $q_{i}$ be the amount of the resource that $i$ actually consumes during $P$, then the amount that $i$ pays per second is

$$
s_{i}=\min \left(\frac{q_{i}}{r_{i}}, 1\right) \frac{b_{i}}{t_{i}} .
$$

This allows users who do not use their full allocation to pay less than their bid, but in no case will a user pay more than his bid.

There are a variety of implementation details. First, the auctioneer gets the number of cycles used by each user from the kernel to determine if $q_{i}<r_{i}$. Second, we set $P=10 \mathrm{~s}$, so the auctioneer charges users and recomputes their bids every 10 seconds. This value is a compromise between the overhead of running the auctioneer and the latency in changing the auctioneer's allocation. With tighter integration with the kernel and the virtualization system, $P$ could be as small as the scheduling interval $(10 \mathrm{~ms}$ on most systems). Third, users whose bids are too small relative to the other users are logged off the system. Users who bid for less than .1\% of the resource would run infrequently while still consuming overhead for context-switching, accounting, etc., so the auctioneer logs them off, starting with the smallest bidder.

The advantages of this allocation function 3.3 .3 are that it is simple, it can be computed in $O(n)$ time, where $n$ is the number of bidders, it is fair, and it can be optimized across multiple auctioneers by an agent (described in $\S$ 3.4). It is fair in the sense that all users who use their entire allocation pay the same per unit of the resource.

The disadvantage is that it is not strategyproof. In the simple case of one user running on a host, that user's best (or dominant) strategy is to make the smallest possible bid, which would still provide the entire host's resources. If there are multiple users, then the user's dominant strategy is to bid his valuation. Since, the user's dominant strategy depends 
on the actions of others, this mechanism is not strategyproof. One possible strategyproof mechanism is a Generalized Vickrey Auction (GVA) [30. However, this requires $O\left(n^{2}\right)$ time, it is not fair in the sense described above, and it is not clear how to optimize bidding across multiple GVA auctioneers.

\subsubsection{Advertising Availability}

The auctioneer must advertise the availability of local resources so that user agents can decide whether to place bids. For each resource available on the local host, the auctioneer advertises the total amount available, and the total amount spent at the last allocation. In other words, the auctioneer reports

$$
\sum_{j=0}^{n-1} s_{i} .
$$

This may be less than the sum of the bids because some tasks did not use their entire allocation. We report this instead of the sum of the bids because it allows the agent to more accurately predict the cost of resources (as required the algorithm described in $\S 3.4 .1$. Note that this information allows agents to make appropriate bids without revealing the exact amounts of other users' individual bids. Revealing that information would allow users to know each other's valuations, which would allow gaming the auctions.

\subsection{Agent}

The role of a tycoon agent is to interpret a user's preferences, examine the state of the system, make bids appropriately, and verify that the resources were provided. The agent is involved in steps 2,3 , 4 , and 6 of Figure 1 Given the diversity of possible preferences, we chose to separate agents from the infrastructure to allow agents to evolve independently. This is a similar approach to the end-to-end principle used in the design of the Internet [8, 11, 24, where application-specific functionality is contained in the end-points instead of in the infrastructure. This allows the infrastructure to be efficient, while supporting a wide variety of applications.

There are a wide variety of preferences that a user can specify to his agent. Tycoon provides for both high-level preferences that an agent interprets and low-level preferences that users must specify in detail. Examples of high level preferences are wanting to maximize the expected number of CPU cycles or to seek machines with a minimum amount of memory, or some combination of those preferences.
Tycoon allows uncertainty in the exact amount of resource received because other applications on the same host may not use their allocation and/or other users may change their bids.

\subsubsection{Best Response Algorithm}

In a system with many machines, it is very difficult for users to bid on individual machines to maximize their utilization of the system. In Tycoon, we allow the user to only specify the total bids, or the budget, he is willing to spend and let the agent compute the bids on the machines to maximize the user's utility. In order to compute the optimum bids, the agent must first know the user's utility as a function of the fraction of the machines assigned to the user. Since it is difficult, if not impossible, to figure out the exact formulation of the utility function, we assume a linear utility function for each user. That is, each user specifies a non-negative weight for each machine to express his preference of the machine. Such a weight is chosen by the user and determined mainly by two factors: the system configuration and the user's need. They may vary from user to user. For example, one user may have higher weight on machine $A$ because it has more memory, and another user may have higher weight on $B$ because it has a faster CPU. The weights are kept private to the users.

Now, suppose that there are $n$ machines, and a user has weight $w_{i}$ on machine $i$ for $1 \leq i \leq n$. If the user gets fraction $r_{i}$ from machine $i$, then his utility is

$$
U=\sum_{i=1}^{n} w_{i} r_{i}
$$

The agent's goal is to maximize the user's utility under a given budget, say $X$, and the others' aggregated bids on the machines. Suppose that $y_{i}$ is the total bid by other users on machine $i$. The user's share on $i$ is then $\frac{x_{i}}{x_{i}+y_{i}}$ if he bids $x_{i}$ on machine $i$. Therefore, the agent needs to solve the following optimization problem:

$$
\begin{gathered}
\operatorname{maximize} \sum_{i=1}^{n} w_{i} \frac{x_{i}}{x_{i}+y_{i}}, \quad \text { s.t. } \\
x_{i} \geq 0, \quad \text { for } 1 \leq i \leq n, \text { and } \\
\sum_{i=1}^{n} x_{i}=X .
\end{gathered}
$$

This optimization problem can be solved by using the following algorithm. 
1. sort $\frac{w_{i}}{y_{i}}$ in decreasing order, and suppose that

$$
\frac{w_{1}}{y_{1}} \geq \frac{w_{2}}{y_{2}} \geq \cdots \geq \frac{w_{n}}{y_{n}} .
$$

2. compute the largest $k$ such that

$$
\frac{\sqrt{w_{k} y_{k}}}{\sum_{j=1}^{k} \sqrt{w_{j} y_{j}}}\left(X+\sum_{j=1}^{k} y_{j}\right)-y_{k} \geq 0 .
$$

3. set $x_{i}=0$ for $i>k$, and for $1 \leq i \leq k$, set

$$
x_{i}=\frac{\sqrt{w_{i} y_{i}}}{\sum_{j=1}^{k} \sqrt{w_{j} y_{j}}}\left(X+\sum_{j=1}^{k} y_{j}\right)-y_{i} .
$$

The above algorithm takes $O(n \log n)$ time as sorting is the most expensive step. It is derived by using Lagrangian multiplier method. Intuitively, the optimum is achieved by the bids where the bid on each machine has the same marginal value. The challenge is to select the machines to bid on. Roughly speaking, one should prefer to bid on a machine if it has high weight on the machine and if other's bids on that machine is low. That is the intuition behind the first sorting step. We omit the correctness proof of the algorithm due to the space limitation.

One problem with the above algorithm is that it spends the entire budget. In the situation when there are already heavy bids on the machines, it might be wise to save the money for later use. To deal with the problem, a variation is to also prescribe a threshold $\lambda$ to the agent and require that the margin on each machine is not lower than $\lambda$, in addition to the budget constraint. Such problem can be solved by an easy adaptation of the algorithm.

\subsubsection{Predictability}

Instead of maximizing its expected value, some applications may prefer to maintain a minimum amount of a resource. An example of this is memory, where an application will swap pages to disk if it has less physical memory than some minimum, but few applications benefit significantly from having more than that. Tycoon allows agents to express this preference by putting larger bids on fewer machines. Let $R$ be the total resource size on a host and $B$ be the sum of the users' bids for the resource, excluding user $i$. From (3.3.3), the user $i$ 's agent can compute that to get $r_{i}$ of a resource, it should bid

$$
b_{i}=\frac{r_{i} B}{R-r_{i}} .
$$

However, this only provides an expected amount of $r_{i}$. To provide higher assurances of having this amount, the agent bids more than $b_{i}$. To determine how much more, the agent maintains a history of the bids at that host to determine the likelihood that a particular bid will result in obtaining the required amount of a resource. Assuming that the application only uses $r_{i}$ of the resource, the user will pay more per unit of the resource than if his agent had just bid $b_{i}$ (see $\S 3.3 .3$ ), but that is the price of having more predictability.

\subsubsection{Scalability}

Since the computational overhead of the agent is low, the main scalability concern is communications overhead. When making bids, a user agent may have to contact a large number of auctioneers, possibly resulting in a large queueing delay. For example, to use 100 hosts, the agent must send 100 messages. Although the delay to do this is proportional to the amount of resources the user is using, for very large numbers of hosts and a slow and/or poorly connected agent host, the delay may be excessive. In this case, the agent can use an application-layer multicast service (e.g., Bullet 17]) to reduce the delay. Since changing a bid consists of simply setting an interval, the user agent can use a multicast service to send out the same interval to multiple auctioneers. This would essentially make the communication delays logarithmic with respect to number of hosts.

\subsubsection{Verification}

One potential problem with all auction-based systems is that auctioneers may cheat by charging more for resources than the rules of the auction dictate. However, one advantage of Tycoon is that it is market-based so users will eventually find more cost-effective auctioneers. Cost-effectiveness is an application-specific metric. For example, an application may prefer a slow host because it has a favorable network location. Users who are interested in $\mathrm{CPU}$ cycles would view that as a host with poor cost-effectiveness. However, in many applications, the agent can measure cost-effectiveness fairly accurately. As an example, the rendering application we use in $\S$ प uses frames rendered per second as its utility metric. As a result, the cost-effectiveness is frames rendered per second per credit spent for each host.

The measured cost-effectiveness is then used as the host weight for the best-response algorithm. This algorithm will automatically drop a host from bidding when it sees that it is significantly less cost- 
effective than the others. Effectively, Tycoon treats a cheating host as a host with poor cost-effectiveness. Therefore we do need sophisticated techniques to detect or prevent cheating. If no agents wants to spend credits at a cheating auctioneer, the monetary incentive to cheat is greatly reduced.

\subsection{Funding Policy}

Funding policy determines how users obtain funds. We define open loop and closed loop funding policies. In an open loop funding policy, users are funded at some regular rate. The system administrators set their income rate based on exogenously determined priorities. Providers accumulate funds and return them to the system administrators. In a closed loop (or peer-to-peer) funding policy, users themselves bring resources to the system when they join. They receive an initial allotment of funds, but they do not receive funding grants after joining. Instead, they must earn funds by enticing other users to pay for their resources. A closed loop funding policy is preferable because it encourages service providers to provide desirable resources and therefore should result in higher economic efficiency.

\section{Experiments}

\subsection{Experimental Setup}

The experiments in this section were run the on the hosts shown in Table 4.1 A were running Linux with the PlanetLab 2.4.22 kernel, which includes VServer and plkmod.

\subsection{Agility}

In this section, we report the results of experiments to test agility, the ability to adapt to changes in demand. As a workload, we used the Maya 6.0 image rendering software to renderer frames in a movie scene. The jobs were dispatched using the Muster job queue, an off-the-shelf product that manages distributed rendering jobs. During the experiment two users were rendering concurrently on each node.

First, we examine the time for a user to acquire more resources to finish his rendering job sooner. In Figure 2 a user has initialized his nodes with $\$ 10$ to be spent over 30,000 seconds. He submits a 200 frame rendering job to the Tycoon cluster. Someone else is already running on the cluster. Using the bids of both users, auctioneers allocate the new user about twenty percent of each node. After running for three minutes, the user notices that the job is not likely

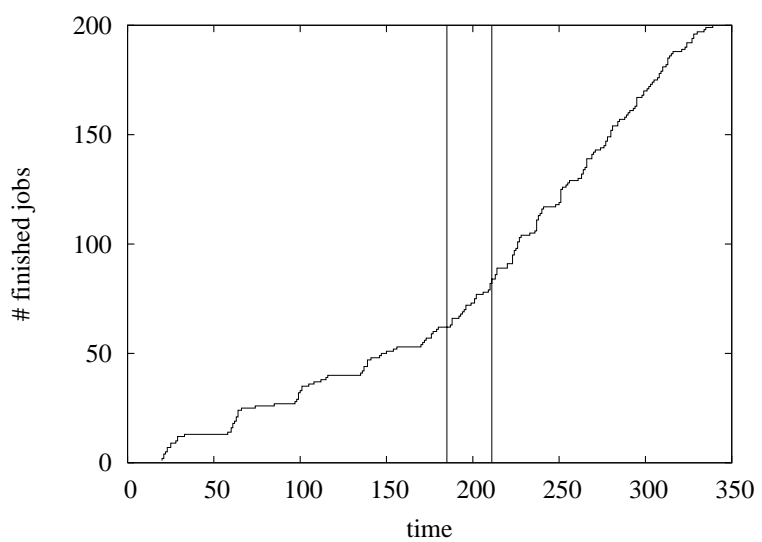

Figure 2: This figure shows a user increasing his share at 190 seconds by decreasing the bidding interval. As a result, the throughput increases by 210 seconds.

to finish early enough, so he changes the spending interval to 300 seconds on all nodes. This will leave him with fewer credits at the end of the run than if he left the interval at 30,000, but it is worth it to him. The time at when he changes the interval is marked by the left vertical line. About twenty seconds later, the right vertical line marks the time at which the user is able to detect an increased rate of rendering. Afterward, the frames finish at an increased speed, and the job finishes on time.

This demonstrates the system's ability to quickly reallocate resources. As in this case, this could be because a user cannot accurately estimate the resource requirements of his application. Other possible causes are that hosts have failed, the load has increased, the user's deadline has changed, etc. The agility of the system allows users to compensate for uncertainty.

In a second experiment, we examine the system's ability to change allocations when a high priority job is started. In this scenario, two users are rendering on the cluster. One user performs a low priority render, and he funds his nodes with $\$ 10$ for 100,000 seconds. A second user funds his nodes with $\$ 10$ for 10,000 seconds. Initially, only the low priority job is running, but after 220 seconds, the second user submits a rendering job to the system.

Figure 3 shows the average rate at which frames are finished for the two jobs. First the low priority job runs alone, at an average rate of 1.1 frames per second. When second users submits the high priority job, (marked with a vertical line in the figure), the throughput of the low priority job decreases almost immediately to 0.2 frames per second, and the high priority job starts to render at 0.9 frames per second. 


\begin{tabular}{|c|c|c|c|c|c|}
\hline Processor Variety & CPU & Memory & Disk & \# nodes & Location \\
\hline \hline Pentium III & $1 \mathrm{GHz}$ & $2 \mathrm{~GB}$ & 32 GB SCSI & 4 & US \\
\hline Mobile Pentium III & $900 \mathrm{MHz}$ & $512 \mathrm{MB}$ & $40 \mathrm{~GB}$ IDE & 8 & UK \\
\hline Pentium III & $550 \mathrm{MHz}$ & $256 \mathrm{MB}$ & $10 \mathrm{~GB}$ IDE & 2 & US \\
\hline Pentium II & $450 \mathrm{MHz}$ & $128 \mathrm{MB}$ & $10 \mathrm{~GB}$ IDE & 6 & UK \\
\hline
\end{tabular}

Table 2: Specifications of the four types of computers used in the test cluster.

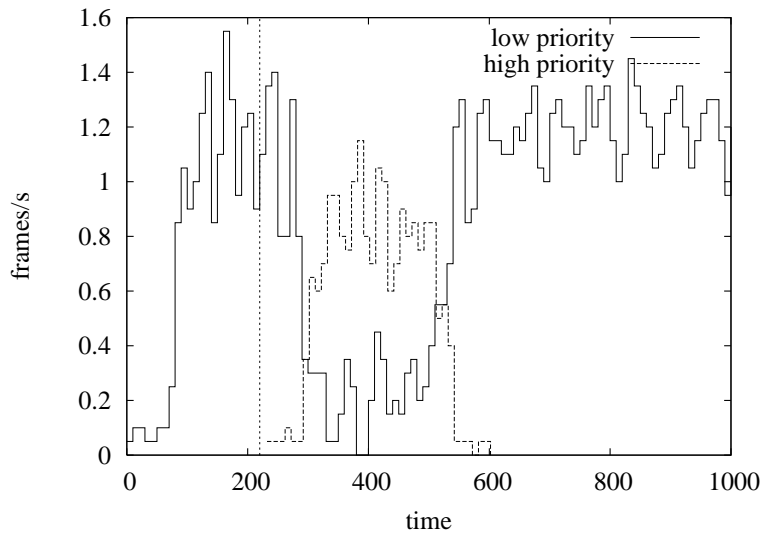

Figure 3: This figure shows a low priority job with a small share getting lower throughput when a high priority job arrives.

As soon as the high priority job finishes, the low priority job starts to utilize the CPUs again, and gets an increased throughput.

When high priority job first starts, it has lower throughput because it is waiting for disk I/O. During that time, the low priority job is able to continue to utilize the CPU. As soon as the high priority job is ready to run, it produces frames at almost full speed. Based on the bids, its share is $90 \%$. The actual throughput is on average $0.9 / 1.1$, which is slightly lower. This is also because of disk I/O delays. The throughput penalty from $\mathrm{I} / \mathrm{O}$ is higher for the high priority task than for the low priority task because it issues more I/O operations. This is an artifact of our version of VServer being unable to regulate disk I/O bandwidth. If our virtualization layer had that capability, the actual throughput would be closer to the ideal of $90 \%$.

In the third experiment, we show how the system treats a user who runs infrequently in comparison to one that runs continously. Both users initialize their nodes with $\$ 10$ for 300 seconds. One user starts a long continuous job on the cluster. While the user is running alone, his share decreases in proportion to $\left(1-P / t_{i}\right)^{\tau / P}$ where $\tau$ is the time since the start of the experiment, $t_{i}=300$ is the funding interval, and

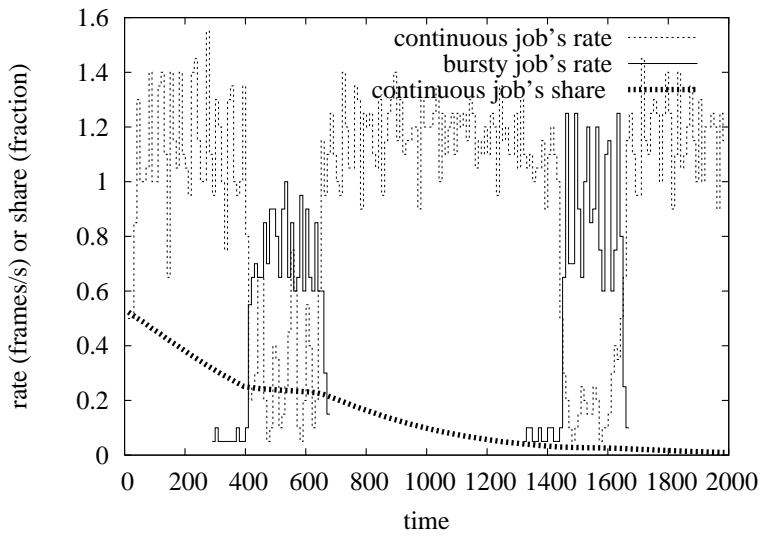

Figure 4: This figure shows how a user that runs infrequently can receive more resources when he does run in comparison to a user that runs continuously.

$P=10$ is the auctioneers' update interval. Since the infrequent user is not running, the continuous job initially gets to use the whole cluster, as shown in Figure 4

After 400 seconds, the infrequent user starts running. Since it has not spent any money, it's share is 75 percent, and the job that has been running has a 25 percent share. Since both jobs continue to pay in proportion to their balance, their shares remain at 75 and 25 percent, respectively, until the infrequnt users stops running. The infrequent user returns at 1300 seconds and again he gets most of the resources. In this case, he gets most of the resources because the continuous user's share has dropped considerably.

The key point about this result is that the system encourages efficient usage of resources even when users do not make explicit bids. In this experiment, the users bids were identical, which could have been set when their accounts were created. Despite this, the infrequent user is rewarded for being judicious in his resource consumption, while the continuous user is penalized for running all the time. In comparison, a proportional share system would allocate $50 \%$ of the resources to each user when both are running. This gives no disincentive for the continuous user to stop running. The performance improvement for 


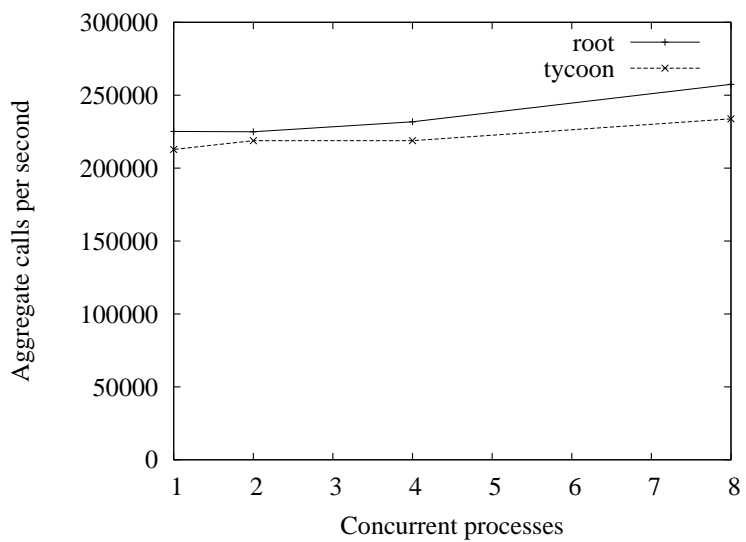

Figure 5: System Call Performance

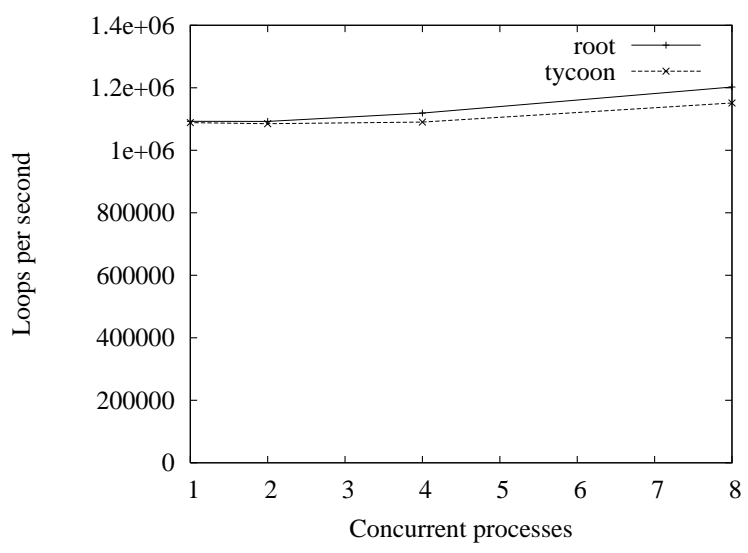

Figure 6: CPU-bound Task Performance

the infrequent user is $(.75-.50) / .50=50 \%$ for one continuous user. For $n$ continuous users, the performance improvement is $(.75-(1 / n)) /(1 / n)$, which goes to infinity as $n$ goes to infinity.

\subsection{Host Overhead}

This set of experiments measures the overhead incurred by using Tycoon rather than using the same Linux computer without Tycoon. This overhead includes VServer, plkmod, and the auctioneer overhead. We compared this relative performance for three distinct types of operations. They are illustrated in Figures 5 6 7 and 8 for system call overhead, CPU-bound computation, disk reading and disk writing, respectively. In these experiments, from one to eight programs designed to test a particular type of operation are invoked simultaneously by ssh. For the Tycoon experiments, each program is started as a distinct user. In the root scenario, the programs are all run as root. The sum of the scores of all of

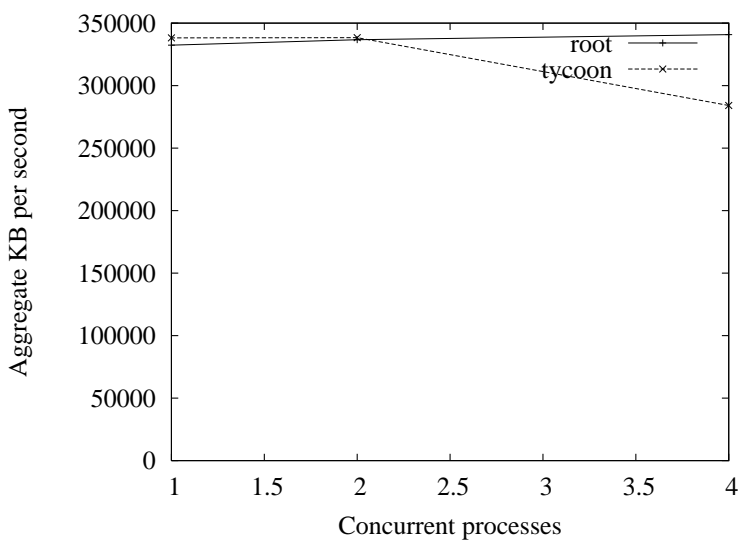

Figure 7: Disk Read Performance

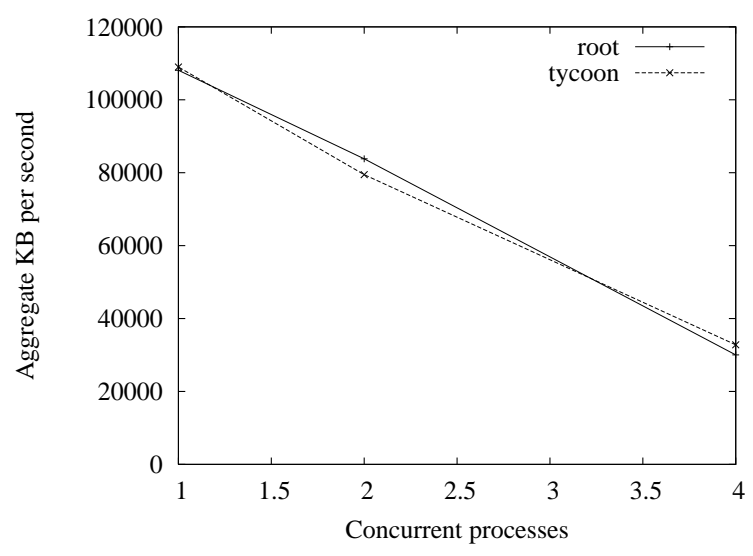

Figure 8: Disk Write Performance 


\begin{tabular}{|l|l|l|l|l|}
\hline Operation & SLS & Bank & Auc. & Agent \\
\hline \hline Registration (per min.) & 260 & & 9634 & \\
\hline SLS query (20 hosts) & $89 \mathrm{~K}$ & & & 311 \\
\hline Bank transfer & & 1198 & & 610 \\
\hline Account creation & & & 5901 & 3592 \\
\hline Spending rate change & & & 793 & 719 \\
\hline
\end{tabular}

Table 3: Bytes sent from the specified entity while conducting the specified operation.

the concurrent processes is plotted as a function of the number of concurrent processes.

For CPU-bound processes and for a few I/Obound processes, Tycoon has less than five percent overhead. We expect the bulk of cluster applications to be similar to these micro-benchmarks. For processes that involve many system calls, the overhead is capped at ten percent, but we do not expect many Tycoon processes to be system call-heavy. The overhead for Tycoon is most significant for many disk reading processes. This may be due to the additional memory overhead of VServer reducing the size of the buffer cache, but we are still investigating this.

\subsection{Network Overhead}

The primary bottlenecks that prevent the unfettered scaling of a Tycoon cluster are the two centralized servers, the service location server (SLS) and the bank. Table 3 quantifies the costs of performing the most common operations on a Tycoon cluster.

The most frequent process is the maintenance of soft-state between the auctioneers and the SLS. Assuming that the SLS is allowed to use $100 \mathrm{Mb} / \mathrm{s}$ network bandwidth (e.g., it is on a $1 \mathrm{~Gb} / \mathrm{s}$ network), it can manage up to 75,000 Tycoon hosts. If clients use the best response agent to operate on the Tycoon cluster, they must issue repeated host-list queries to the SLS to compute their optimal bidding strategy. If the agent updates its strategy once a minute, it costs roughly $4 \mathrm{~KB} /$ minute per agent per host. Again assuming this task is allocated $100 \mathrm{Mb} / \mathrm{s}$ of bandwidth, the product of the number of agents and number of hosts must not exceed 187M. Hence assuming that there are $75 \mathrm{~K}$ hosts in the cluster, there may be up to 2500 agents running concurrently. Similarly if there are only 2500 hosts, there may be up to $75 \mathrm{~K}$ agents.

A less frequent operation is bank transfers from users to hosts. This task depends less on bandwidth and more on the speed of the bank system in performing large integer arithmetic for authentication. On a $450 \mathrm{MHz}$ Pentium III, this operation requires an average of $100 \mathrm{~ms}$. Assuming user perform bank operations every twenty minutes per user per host, this bank supports an active user-host product of 12,000 , which would allow 24 simultaneous active users on a 500 host cluster. As a result, for the immediate future, we do not believe a centralized bank is a significant problem. One reason is that much faster hardware is available. A $3 \mathrm{GHz}$ bank should support 6.7 times the number of users or hosts or combination thereof. Another reason is that the current protocol performs only one credit transfer per connection. It could be optimized to perform multiple transfers per connection which would amortize the authentication and communication costs. Finally, twenty minutes is a very conservative estimate of bank operations. A more likely frequency is once a day. This would allow even the current slow hardware and unoptimized protocol to support a userhost product of 864000 . A centralized bank is not likely to limit scalability in practice.

\section{Related Work}

In this section, we describe related work in resource allocation. There are two main groups: those that incorporate an economic mechanism ${ }^{1}$, and those that do not.

One of the key non-economic abstractions for resource allocation is a computer science context is Proportional Share (PS), originally documented by Tijdeman 28. Each PS process $i$ has a weight $w_{i}$. The share of a resource that process $i$ receives over some interval $t$ where $n$ processes are running is

$$
\frac{w_{i}}{\sum_{j=0}^{n-1} w_{j}}
$$

PS maximizes utilization because it always provides resources to needy processes. One problem is that PS is usually applied by giving each user a weight and directly transferring that weight to the user's processes. However, a user may not weigh all of his processes equally and PS does not give an incentive for users to differentiate his processes. As a result, as a system becomes more loaded, the low value processes consume more resources, until the high value processes cannot make useful progress (as shown by Lai, et al. [18]).

One common method for dealing with this problem is to rely on social mechanisms to set the PS weights appropriately. A system administrator could set them based on input from users or users could

\footnotetext{
${ }^{1}$ By mechanism we mean the system that provides an incentive for users to reveal the truth (e.g., an auction)
} 
"horse trade" high weights amongst themselves. Although these mechanisms work well for small groups of people that trust each other, they do not scale to larger groups and they have a high overhead in user time.

Most recent work by Waldspurger and Weihl 32, Stoica, et al. 25], and Nieh, et al. 20] on PS has focused on computationally efficient and fair implementations. Lottery scheduling 32 is a PS-based abstraction that is similar to the economic approach in that processes are issued tickets that represent their allocations. Sullivan and Seltzer 27] extend this to allow processes to barter these tickets. Although this work provides the software infrastructure for an economic mechanism it does not provide the mechanism itself.

Similarly, SHARP (described by Fu, et al. 14]) provides the distributed infrastructure to manage tickets, but not the mechanism or agent strategies. In addition, SHARP and work by Urgaonkar, et al. [29] use an overbooking resource abstraction instead of PS. An overbooking system promises probabilistic resources to applications. Tycoon uses a similar abstraction for applications that require a minimum amount of a resource.

Another class of non-economic algorithms examine resource allocation from a scheduling (surveyed by Pindedo 23] ) perspective using combinatorial optimization (described by Papadimitriou and Steiglitz [22]) or by examining the resource consumption of tasks (a recent example is work by Wierman and Harchol-Balter 34]). However, these assume that the values and resource consumption of tasks are reported accurately. This assumption does not apply in the presense of strategic users. We view scheduling and resource allocation as two separate functions. Resource allocation divides a resource among different users while scheduling takes a given allocation and orders a user's jobs.

Examples the economic approach are Spawn (by Waldspurger, et al. 31]), work by Stoica, et al. 26. ., the Millennium resource allocator (by Chun, et al. [10]), work by Wellman, et al. 33, and Bellagio (by AuYoung, et al. [5]).

Spawn and the work by Wellman, et al. uses a reservation abstraction similar to the way airline seats are allocated. Although reservations allow low risk, the utilization is also low because some tasks do not use their entire reservations. Service applications (e.g., web serving, database serving, and overlay network routing) result in particularly low utilization because they typically have bursty and unpredictable loads. Another problem with reservations is that they can significantly increase the latency to ac- quire resources. A reservation by one user prevents another user from using the resources for the duration of the reservation, even if the new user is willing to pay much more for the resources than the first user. Reservations are typically on the order of minutes or hours (Spawn used 15 minutes), which is too much delay for a highly bursty and unpredictable application like web serving.

The proportional share abstraction used in the Millennium resource allocator comes the closest to that used in Tycoon. We extend that abstraction with continuous bids, the best-response agent algorithm, and secure protocols for bidding.

Bellagio uses a centralized allocator called SHARE developed by Chun, et al. 9]. SHARE takes the combinatorial auction approach to resource allocation. This allows users to express preferences with complementarities like wanting host $\mathrm{A}$ and host $\mathrm{B}$, but not wanting host $\mathrm{A}$ without $\mathrm{B}$ or $\mathrm{B}$ without $\mathrm{A}$. The combinatorial auction approach relies on a centralized auctioneer to guarantee that the user either gets both $\mathrm{A}$ and $\mathrm{B}$ or else nothing. Economic theory predicts that solving this NP-complete problem provides an allocation with optimal economic efficiency. Tycoon addresses the combinatorial problem in a possibly less economically efficient, but more scalable way. In Tycoon, credits are only spent when the user actually consumes resources, so the user's agent can see that it only has A before his application runs and thereby prevent wasting credits on an unvalued resource. The disadvantages of the combinatorial auction approach are the centralized auctioneer and the difficulty of the combinatorial auction problem. The centralized auctioneer is vulnerable to compromise and limits the scalability of the system, especially since it must be involved in all allocations. Moreover, even computationally efficient heuristic algorithms operate on the order of minutes, while Tycoon reallocates in less than ten seconds. Recent work by Hajiaghayi [15] on online resource allocation may be able to reduce the delay of the combinatorial approach.

\section{Future Work}

One area of future work is more complete virtualization. Our prototype implementation uses early versions of VServer and plkmod which only support virtualization of CPU cycles. Later versions of VServer, Xen [12, and the Class-based Kernel Resource Management (CKRM) 4] support more complete virtualization and should be relatively straight-forward to integrate with Tycoon.

Another area of future work is to develop a scal- 
able banking infrastructure. One possibility is to physically distribute the bank without administratively distributing it. The bank would consist of several servers with independent account databases. A user has accounts on some subset of the servers. A user's balance is split into separate balances on each server. To make a transfer, users find a server where both the payer and payee have an account and that contains enough funds. The transfer proceeds as with a centralized bank. Users should periodically redistribute their funds among the servers to ensure that one server failure will not prevent all payment.

\section{Summary}

An economic mechanism is vital for large-scale resource allocation. In this paper, we propose a distributed market where auctioneers only manage local resources. A user's agent sends separate bids to these auctioneers, where each bid is for a single type of resource at that host. The bids are continuous bids in that they stay in effect until the user's local balance is depeleted. Resources are allocated to users in proportion to their bids using a best-effort model. Agents are responsible for optimizing their users' utility.

Using our prototype implementation, we show: 1) continuous bids reduce the burden on users by allowing them to run without frequent interactive bidding while still making an efficient and low-latency allocation; 2) distributed auctioneers result in very low overhead for allocation; and 3) the best-response algorithm can optimize across multiple markets.

\section{Acknowledgements}

Several people provided key contributions without which this research would not have been possible. David Connell assembled, installed, and administered the Tycoon cluster. Peter Toft provided the machines in Bristol and John Henriksson maintained them. John Janakiraman provided several machines in Palo Alto. The economic mechanisms described here benefited from several discussions with Leslie Fine.

\section{References}

[1] http://planet-lab.org.

[2] http://www.linux-vserver.org/.

[3] http://www.vmware.com/.

[4] 2004. http://ckrm.sourceforge.net/.
[5] AuYoung, A., Chun, B. N., Snoeren, A. C., AND VAhdat, A. Resource Allocation in Federated Distributed Computing Infrastructures. In Proceedings of the 1st Workshop on Operating System and Architectural Support for the On-demand IT InfraStructure (2004).

[6] Bavier, A., Bowman, M., Chun, B., Culler, D., Karlin, S., Muir, S., Peterson, L., Roscoe, T., Spalink, T., AND WaWrzoniak, M. Operating System Support for Planetary-Scale Network Services. In Symposium on Networked Systems Design and Implementation (2004).

[7] Bugnion, E., Devine, S., Govil, K., And RosenBLum, M. Disco: Running Commodity Operating Systems on Scalable Multiprocessors. ACM Transactions on Computer Systems 15, 4 (1997), 412-447.

[8] Cerf, V., And Kahn, R. A Protocol for Packet Network Intercommunication. IEEE Transactions on Computers 22, 5 (May 1974), 637-648.

[9] Chun, B., Ng, C., Albrecht, J., Parkes, D. C., And VAhdat, A. Computational Resource Exchanges for Distributed Resource Allocation. 2004.

[10] Chun, B. N., And Culler, D. E. Market-based Proportional Resource Sharing for Clusters. Technical Report CSD-1092, University of California at Berkeley, Computer Science Division, January 2000.

[11] Clark, D. D. The Design Philosophy of the DARPA Internet Protocols. In SIGCOMM (1988), pp. 106-114.

[12] Dragovic, B., Fraser, K., Hand, S., Harris, T., Ho, A., Pratt, I., Warfield, A., Barham, P., And Neugebauer, R. Xen and the Art of Virtualization. In Proceedings of the ACM Symposium on Operating Systems Principles (2003).

[13] Foster, I., And Kesselman, C. Globus: A Metacomputing Infrastructure Toolkit. The International Journal of Supercomputer Applications and High Performance Computing 11, 2 (Summer 1997), 115-128.

[14] Fu, Y., Chase, J., Chun, B., Schwab, S., And VAHDAT, A. SHARP: An Architecture for Secure Resource Peering. In ACM Symposium on Operating Systems Principles (SOSP) (October 2003).

[15] Hajiaghayi, M. T., Kleinberg, R., and Parkes, D. C. Adaptive Limited-Supply Online Auctions. In Proc. ACM Conf. on Electronic Commerce (2004), pp. $71-80$.

[16] Hurwicz, L. The Design of Mechanisms for Resource Allocation. American Economic Review Papers and Proceedings 63 (1973), 1-30.

[17] Kostic, D., Rodriguez, A., Albrecht, J., And VAhdat, A. Bullet: High Bandwidth Data Dissemination Using an Overlay Mesh. In Proceedings of the 19th ACM Symposium on Operating System Principles (2003). 
[18] Lai, K., Huberman, B. A., And Fine, L. Tycoon: A Distributed Market-based Resource Allocation System. Tech. rep., arXiv, 2004. http://arxiv.org/abs/cs.DC/0404013

[19] Massie, M. L., Chun, B. N., And Culler, D. E. The Ganglia Distributed Monitoring System: Design, Implementation, and Experience. Parallel Computing 30, 7 (July 2004).

[20] Nieh, J., Vaill, C., And Zhong, H. Virtual-Time Round-Robin: An O(1) Proportional Share Scheduler. In Proceedings of the USENIX Annual Technical Conference (2001).

[21] Oppenheimer, D., Albrecht, J., Patterson, D., And Vahdat, A. Scalable Wide-Area Resource Discovery. Tech. rep., U.C. Berkeley, July 2004.

[22] Papadimitriou, C. H., And Steiglitz, K. Combinatorial Optimization. Dover Publications, Inc., 1982.

[23] Pinedo, M. Scheduling. Prentice Hall, 2002.

[24] Saltzer, J. H., Reed, D. P., And Clark, D. D. End-To-End Arguments in System Design. ACM Transactions on Computer Systems 2, 4 (1984), 277-288.

[25] Stoica, I., Abdel-Wahab, H., Jeffay, K., Baruah, S., Gehrke, J., and Plaxton, G. C. A Proportional Share Resource Allocation Algorithm for Real-Time, Time-Shared Systems. In IEEE Real-Time Systems Symposium (December 1996).

[26] Stoica, I., Abdel-Wahab, H., And Pothen, A. A Microeconomic Scheduler for Parallel Computers. In Proceedings of the Workshop on Job Scheduling Strategies for Parallel Processing (April 1995), pp. 122-135.

[27] Sullivan, D. G., And Seltzer, M. I. Isolation with Flexibility: a Resource Management Framework for Central Servers. In Proceedings of the USENIX Annual Technical Conference (2000), pp. 337-350.

[28] Tijdeman, R. The Chairman Assignment Problem. Discrete Mathematica 32 (1980).

[29] Urgaonkar, B., Shenoy, P., and Roscoe, T. Resource Overbooking and Application Profiling in Shared Hosting Platforms. In Proceedings of Operating Systems Design and Implementation (December 2002).

[30] Varian, H. R. Economic Mechanism Design for Computerized Agents. In Proc. of Usenix Workshop on Electronic Commerce (July 1995).

[31] Waldspurger, C. A., Hogg, T., Huberman, B. A., Kephart, J. O., and Stornetta, W. S. Spawn: A Distributed Computational Economy. Software Engineering 18, 2 (1992), 103-117.

[32] Waldspurger, C. A., And Weihl, W. E. Lottery Scheduling: Flexible Proportional-Share Resource Management. In Operating Systems Design and Implementation (1994), pp. 1-11.
[33] Wellman, M. P., Walsh, W. E., Wurman, P. R., and MacKie-Mason, J. K. Auction Protocols for Decentralized Scheduling. Games and Economic Behavior 35 (2001), 271-303.

[34] Wierman, A., and Harchol-Balter, M. Classifying Scheduling Policies with respect to Unfairness in an M/GI/1. In Proceedings of the ACM SIGMETRICS 2003 Conference on Measurement and Modeling of Computer Systems (2003). 